\title{
Generation, annotation, and analysis of ESTs from midgut tissue of adult female Anopheles stephensi mosquitoes
}

Deepak P Patil*1, Santosh Atanur*2, Dhiraj P Dhotre*1, D Anantharam*1, Vineet S Mahajan1, Sandeep A Walujkar1, Rakesh K Chandode1, Girish J Kulkarni ${ }^{1}$, Pankaj S Ghate ${ }^{1}$, Abhishek Srivastav ${ }^{1}$, Kannayakanahalli M Dayananda1, Neha Gupta1, Bhakti Bhagwat ${ }^{2}$, Rajendra R Joshi ${ }^{2}$, Devendra T Mourya ${ }^{3}$, Milind S Patole ${ }^{1}$ and Yogesh S Shouche*1

Address: ${ }^{1}$ Lab 3, National Center for Cell Science, Pune - 411007, India, ${ }^{2}$ Bioinformatics Team, Center for Development of Advanced Computing, Pune University Campus, Pune - 411007, India and ${ }^{3}$ Microbial Containment Complex, National Institute of Virology, Pune - 411007, India

Email: Deepak P Patil* - deepakpatilp@gmail.com; Santosh Atanur* - santoshatanur@gmail.com;

Dhiraj P Dhotre* - dheerajdhotre@gmail.com; D Anantharam* - nirvikalpa@rediffmail.com; Vineet S Mahajan - vineetsm2002@gmail.com; Sandeep A Walujkar - sandeepwalujkar@gmail.com; Rakesh K Chandode - rchandode@gmail.com; Girish J Kulkarni - girishmbu@gmail.com; Pankaj S Ghate - ghate.pankaj@gmail.com; Abhishek Srivastav - seqdgene@gmail.com;

Kannayakanahalli M Dayananda - kmd1234@gmail.com; Neha Gupta - nehagupta279@gmail.com; Bhakti Bhagwat - bhaktib@cdac.in;

Rajendra R Joshi - rajendra@cdac.in; Devendra T Mourya - mouryadt@vsnl.net; Milind S Patole - patole@nccs.res.in;

Yogesh S Shouche* - yogesh@nccs.res.in

* Corresponding authors

Published: 20 August 2009

BMC Genomics 2009, 10:386 doi:10.1186/147/-2164-10-386
Received: 16 September 2008

Accepted: 20 August 2009

This article is available from: http://www.biomedcentral.com/I47/-2/64//0/386

(C) 2009 Patil et al; licensee BioMed Central Ltd.

This is an Open Access article distributed under the terms of the Creative Commons Attribution License (http://creativecommons.org/licenses/by/2.0), which permits unrestricted use, distribution, and reproduction in any medium, provided the original work is properly cited.

\begin{abstract}
Background: Malaria is a tropical disease caused by protozoan parasite, Plasmodium, which is transmitted to humans by various species of female anopheline mosquitoes. Anopheles stephensi is one such major malaria vector in urban parts of the Indian subcontinent. Unlike Anopheles gambiae, an African malaria vector, transcriptome of $A$. stephensi midgut tissue is less explored. We have therefore carried out generation, annotation, and analysis of expressed sequence tags from sugarfed and Plasmodium yoelii infected blood-fed (post $24 \mathrm{~h}$ ) adult female $A$. stephensi midgut tissue.

Results: We obtained $706 \mathrm{I}$ and 8306 ESTs from the sugar-fed and $P$. yoelii infected mosquito midgut tissue libraries, respectively. ESTs from the combined dataset formed 1319 contigs and 2627 singlets, totaling to 3946 unique transcripts. Putative functions were assigned to 1615 (40.9\%) transcripts using BLASTX against UniProtKB database. Amongst unannotated transcripts, we identified 1513 putative novel transcripts and 818 potential untranslated regions (UTRs). Statistical comparison of annotated and unannotated ESTs from the two libraries identified I I 9 differentially regulated genes. Out of 3946 unique transcripts, only 1387 transcripts were mapped on the $A$. gambiae genome. These also included 189 novel transcripts, which were mapped to the unannotated regions of the genome. The EST data is available as ESTDB at http:// mycompdb.bioinfo-portal.cdac.in/cgi-bin/est/index.cgi.
\end{abstract}




\begin{abstract}
Conclusion: 3946 unique transcripts were successfully identified from the adult female $A$. stephensi midgut tissue. These data can be used for microarray development for better understanding of vector-parasite relationship and to study differences or similarities with other malaria vectors. Mapping of putative novel transcripts from $A$. stephensi on the $A$. gambiae genome proved fruitful in identification and annotation of several genes. Failure of some novel transcripts to map on the $A$. gambiae genome indicates existence of substantial genomic dissimilarities between these two potent malaria vectors.
\end{abstract}

\section{Background}

Anopheles stephensi is a major malaria vector in the Indian subcontinent [1]. Rapid urbanization and development in the region has stimulated a corresponding increase in their population resulting in frequent malaria outbreaks [2]. Although, recent malaria epidemics occurred at higher frequencies, mortality was considerably low. For example during 2003, of the reported 1.78 million cases, only 1006 deaths were recorded in India [3].

Absence of an efficient vaccine [4], evolution of drugresistance in the parasites [5], and insecticide-resistance in the mosquitoes [2] accentuate the need of an effective malaria control strategy. Human immunization against parasite proteins through transmission blocking vaccines (TBVs) [6] is one such strategy. Bacterial and fungal-based mosquito control methods are other alternatives but these suffer from major difficulties in practical application $[7,8]$. Transgenic mosquitoes could provide another control method [9], but successful application in field will require designing of appropriate vector-parasite study model [10]. Ito et al. [9], showed transgenic expression of an antiparasitic peptide SM1 in mosquitoes leading to impairment in Plasmodium berghei development. However, the peptide failed to show such activity against Plasmodium falciparum [10,11], the human malaria parasite. Study of naturally occurring $P$. falciparum-resistant A. gambiae mosquitoes revealed a Plasmodium-responsive gene, Anopheles Plasmodium-responsive leucine-rich repeat 1 (APL1) [12], which could form a potent target for the transgenic approach against $P$. falciparum. Many other antiparasitic and/or immunologically active genes like SRPNG [13] from A. gambiae and A. stephensi, TEP1 [14] and leucine-rich repeat protein (LRIM1) [15] from A. gambiae have also been identified recently. Moreover, availability of A. gambiae genome sequence [16] has improved the chances of discovery of more such potential genes in this insect.

In the pre-genomic era, EST (Expressed Sequence Tag) based studies were adopted to understand A. gambiae [1720] and its role in malaria transmission [21]. However, despite its importance as a malaria vector, $A$. stephensi has not been intensively investigated. Although EST [22-24] and microarray-based [25] studies on A. stephensi and
Plasmodium exist, no major transcriptome based contributions have been reported so far. Here, we report the first large-scale effort in construction and analysis of EST libraries from midgut tissue of sugar-fed (SF) and $P$. yoelii infected blood-fed (post 24 h) (BF) female A. stephensi. In light of limited genomic and transcriptomic information for A. stephensi, these data would significantly enrich the molecular aspects of this insect and its role in malaria transmission.

\section{Results}

\section{Generation of ESTs and Pre-processing}

Two cDNA libraries, SF and BF were prepared from sugarfed and $P$. yoelii infected blood-fed (post $24 \mathrm{~h}$ ) adult female A. stephensi mosquito midgut tissues, respectively. Single-pass sequencing yielded 15367 ESTs from both, SF (7061 ESTs) and BF (8306 ESTs) libraries as analyzed by phred [26,27] (quality $\geq 20$ ) with minimum length greater than 100 bases. Vector, adapter, and primer sequences were removed using cross_match [28]. Mouse and Plasmodium sequences were filtered using stand-alone BLAST. Average length of ESTs from both the libraries was approximately 380 bases and approximately $61 \%$ of the sequences were above 300 nucleotides. Table 1 shows the summary of EST data obtained in this study. All the ESTs are deposited in GenBank with continuous accession numbers [GenBank: EX212289 - EX227655].

\section{EST assembly}

CAP3 [29] based EST assembly and clustering of the combined dataset (15367 ESTs) resulted in 1319 contigs and 2627 singlets, forming 3946 unique transcripts (UTs). Similarly, independent assemblies were also performed for SF and BF libraries. Details are given in Table 1.

\section{Assignment of putative functions to ESTs and UTs}

To assign putative function to ESTs and UTs, we performed BLASTX search against the UniProtKB database. Summary of BLASTX results for SF, BF, and combined UTs are shown in Table 2. BLASTX results for combined UTs are given in Additional file 1. Figure 1 shows BLAST hit distribution across various species of organisms for all UTs from the combined dataset. Putative functions were assigned only to 8946 ESTs (58.2\%) and 1615 UTs $(40.9 \%)$ (E-value $\leq 1 e-5)$. Non-coding EST sequences usu- 
Table I: Summary of ESTs generated from adult female $A$. stephensi midgut tissue.

\begin{tabular}{|c|c|c|c|}
\hline Library feature & SF library & BF library & Combined \\
\hline Total ESTs Sequenced (High quality) ${ }^{\dagger}$ & 7061 & 8306 & 15367 \\
\hline Number of Contigs & 822 & 591 & $1319 \ddagger$ \\
\hline Number of ESTs in Contigs & 5243 & 6951 & 12740 \\
\hline Number of Singlets & 1818 & 1355 & 2627 \\
\hline Number of UTs§ & 2640 & 1946 & 3946 \\
\hline Average length of ESTs & 310.69 & 448.90 & $380^{\circ}$ \\
\hline Average length of Contigs & 492.78 & 651.26 & $678.96 \$$ \\
\hline Average \%GC & 45.70 & 52.06 & 48.88 \\
\hline
\end{tabular}

†A high quality sequence is obtained by phred using quality value $\geq 20$ for a stretch 100 bases. $\neq$ Obtained on assembling ESTs from SF and BF libraries. §UTs are calculated as sum of number of contigs and singlets. ${ }^{\circ}$ Obtained using all the ESTs together. \$Calculated using contigs obtained in common assembly of ESTs from both the libraries.

ally fail to find a homolog in the protein databases during BLASTX search [30]. We therefore, screened unannotated (with no BLAST hits) UTs (2331) for the presence of putative coding region using ESTScan program [31]. 1513 UTs were predicted to contain a coding region, thereby suggesting these as novel genes. The remaining sequences could be potential untranslated regions (UTRs). Table 3 shows ESTScan results of both the libraries and combined dataset.

A. stephensi UTs were also compared with the EST sequences of A. gambiae, Aedes aegypti, and Drosophila melanogaster using TBLASTX (E-value $\leq 1 \mathrm{e}-5$ ). As compared to ESTs from Ae. aegypti and D. melanogaster, a higher number of UTs $(45 \%)$ were homologous to A. gambiae ESTs. Only 39\% and 34\% UTs were identified homologous to ESTs from Ae. aegypti and D. melanogaster, respectively (Table 4 ).

\section{Assignment of GO terms \& Statistical Comparison}

Gene Ontology (GO) categories were assigned only to 505,601 , and 629 UTs from the SF, BF, and combined

Table 2: BLAST (BLASTX, E-value $\leq$ le-5) hit summary for UTs against UniprotKB database.

\begin{tabular}{lcc}
\hline Library/dataset & UTs & No. of UTs showing BLAST hit \\
\hline SF & 2640 & 897 \\
\hline BF & 1946 & 1039 \\
\hline Combined & 3946 & 1615 \\
\hline
\end{tabular}

datasets, respectively using Blast2GO program [32]. Additional file 2: Figure S2 illustrates percent similarity and Evalue distribution obtained for all the UTs. Table 5 shows percent distribution of UTs among various assigned GO terms (2nd level) according to the GO consortium [33] for SF and BF libraries. Details of assigned GO terms to each UT from combined dataset are given in Additional file 1. Statistical comparison of GO terms between the two libraries revealed an overrepresentation of metabolic process-related genes in BF library, whereas cellular process-related house keeping genes were dominant in SF library (Figure 2). For details refer Additional file 3.

\section{Statistical comparison of gene expression}

IDEG6 analysis [34] for BLASTX-annotated ESTs identified 114 differentially expressed genes $(P<0.05)$ between the two libraries. In brief, 58 genes ( 20 overexpressed and 38 exclusively expressed) in SF and 56 genes (23 overexpressed and 33 exclusively expressed) in BF library were found to be differentially regulated. Few unannotated genes (unannotated ESTs) were also found to be significantly altered in expression. Two unannotated genes (PU_Contig136 $(P=0)$ and PU_Contig398 $(P=0))$ were exclusively expressed in SF library, while only one such exclusive gene (PI_Contig553 $(P=0)$ ) was found significantly expressed in BF library. Moreover, PU_Contig33 and PI_Contig478 $(P \leq 0.05)$, PU_Contig9 and PI_Contig24 $(P \leq 0.05)$ pairs of unannotated genes were observed in both the libraries with significant changes in gene expression. Many genes were also exclusive to each library but showed no statistical significance. Details are given in Additional file 4. 
Table 3: ESTScan based prediction of potential coding regions in unannotated UTs.

\begin{tabular}{llll}
\hline Number of UTs & SF library & BF library & Combined \\
\hline No. of UTs with no BLAST hit & 1743 & 907 & 2331 \\
\hline No. of UTs as potential UTRs & 620 & 309 & 818 \\
\hline No. of UTs showing a putative coding region & 1123 & 598 & 1513 \\
\hline
\end{tabular}

UTs with no significant hit in UniProtKB database (BLASTX, E-value $\leq l e-5$ ).

\section{Identification of insect-specific transcripts}

To identify insect-specific genes in our UTs, we used data from Zhang et al. [35]. Only 20 transcripts encoding insect-specific proteins were observed in our data and most of them were related to metabolic processes such as reductases and deaminases. A few receptor proteins, sensory and immunity-related proteins were also observed (Additional file 1).

\section{Mapping of ESTs on the Anopheles gambiae genome}

Genome mapping and alignment of A. stephensi UTs on A. gambiae genome revealed many homologous genes between them (Additional file 1). 1387 UTs were successfully mapped on the A. gambiae genome, which also included 189 novel UTs. Remaining UTs (2812) failed to show any alignment with the A. gambiae genome. Table 6 illustrates the details of mapping study.

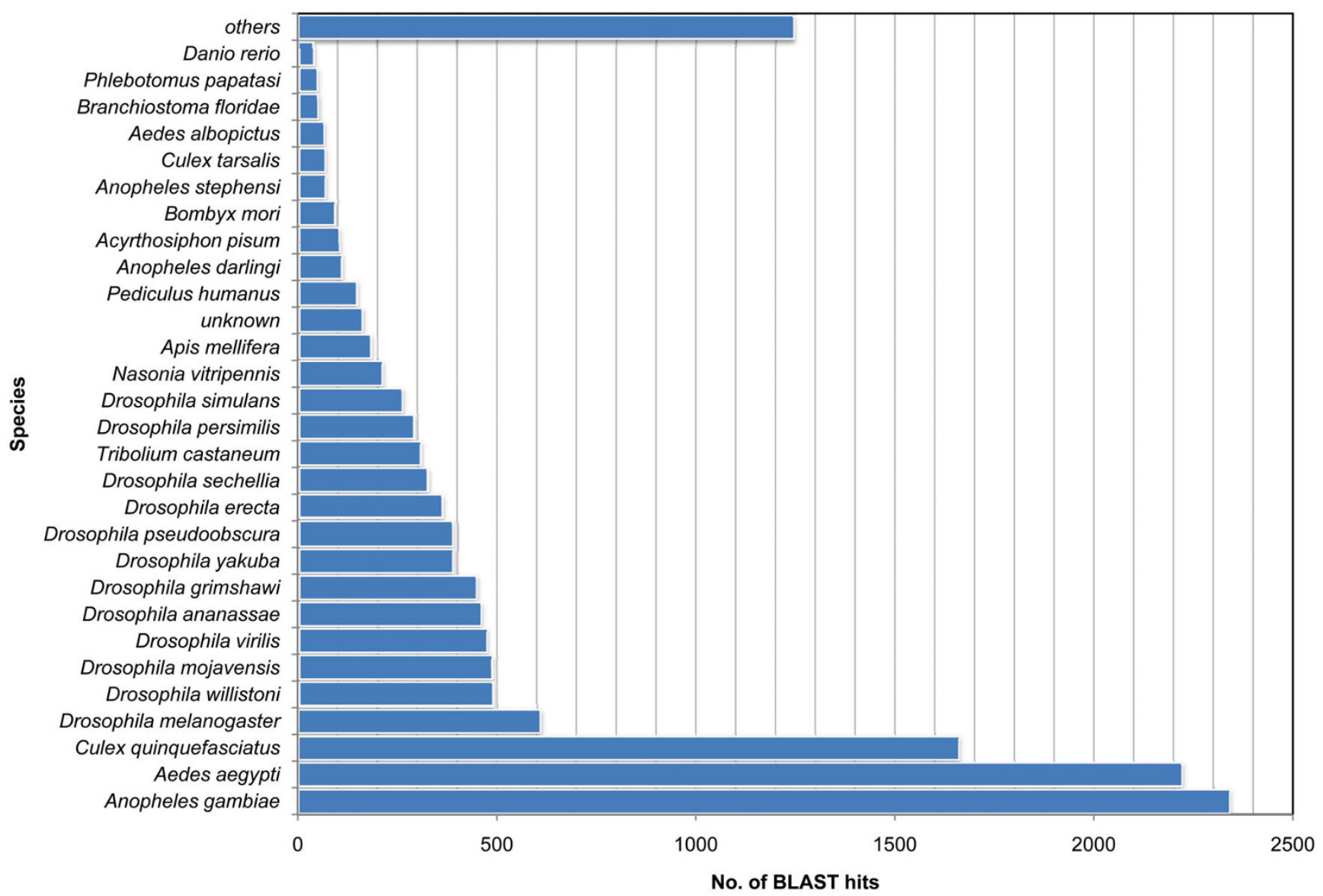

Figure I

BLASTX based species distribution of UTs. Graph generated using Blast2GO program shows species distribution of UTs from combined dataset using BLASTX against non-redundant protein database. Top IO BLAST hits were considered for each transcript. 
Table 4: Number of BLAST (TBLASTX, E-value $\leq$ le-5) hits obtained against three organisms $A$. gambiae, Ae. aegypti, and $D$. melanogaster against ESTdb at NCBI.

\begin{tabular}{lccc}
\hline Library/dataset & \multicolumn{3}{c}{ Organism } \\
\cline { 2 - 4 } & A. gambiae & Ae. aegypti & D. melanogaster \\
\hline SF & 1073 & 863 & 735 \\
\hline BF & 1149 & 1026 & 909 \\
\hline Combined & 1806 & 1544 & 1339 \\
\hline
\end{tabular}

\section{Database Development}

ESTDB http://mycompdb.bioinfo-portal.cdac.in/cgi-bin/ est/index.cgi, a database housing the entire EST dataset along with its annotations has also been developed. The database supports text- and sequence-based queries through a user-friendly interface. It also provides graphical display of contigs along with assembled ESTs.

\section{Discussion}

A. stephensi is a predominant malaria vector in urban parts of the Indian subcontinent. In spite of its importance as a malaria vector, no in-depth transcriptomic information is available on the midgut tissue of $A$. stephensi during sugar feeding and parasite infection. We herein report generation, annotation, and analysis of ESTs from sugar-fed and $P$. yoelii infected adult female A. stephensi midgut tissues.

7061 high quality ESTs were obtained from the sugar-fed cDNA library and 8306 ESTs from the $24 \mathrm{~h}$ post blood-fed infected cDNA library. With 15367 ESTs, our study represents the first intensive effort in complementing gene sequence information for this mosquito. Although the genome of the closely related anopheline species, A. gambiae is available, discovery of novel transcripts (1513) in A. stephensi suggests a significant interspecies variation. In addition, mapping of novel transcripts (189) to the A. gambiae genome testifies the usefulness of our data in gene discovery process.

Like other insects, mosquitoes are also equipped with genes responsible for adaptation to environment changes. Identification of insect-specific genes could prove useful in understanding the molecular basis of their success in various ecological niches. Recently, Zhang et al. [35] identified stress and immune-response related proteins as a major fraction of insect-specific proteins. We have identified 20 such insect-specific genes like reductases, deaminases, receptor proteins, sensory- and immunity-related proteins in this study.

Comparative analysis of GO terms demonstrated striking differences between the two stages of the vector examined.

Table 5: Percent distribution of UTs among the assigned GO terms based on (A) Biological processes, (B) Molecular function, and $(C)$ Cellular component.

GO terms $\quad$ SF library BF library

A) Biological Process

\begin{tabular}{lcc}
\hline Behavior & 0.1 & 0.0 \\
\hline Biological Adhesion & 0.4 & 0.5 \\
\hline Biological Regulation & 4.9 & 4.9 \\
\hline Cellular Process & 39.4 & 39.1 \\
\hline Developmental Process & 5.7 & 4.7 \\
\hline Growth & 0.8 & 0.5 \\
\hline Immune System Process & 0.6 & 0.3 \\
\hline Localization & 7.8 & 7.8 \\
\hline Metabolic Process & 27.4 & 30.0 \\
\hline Multicellular Organismal Process & 6.7 & 6.8 \\
\hline Reproduction & 2.1 & 1.8 \\
\hline Response To Stimulus & 4.0 & 3.5 \\
\hline Rhythmic Process & 0.3 & 0.1 \\
\hline
\end{tabular}

B) Molecular Functions

\begin{tabular}{lcc}
\hline Antioxidant activity & 0.9 & 1.5 \\
\hline Binding & 35.4 & 32.1 \\
\hline Catalytic activity & 33.0 & 39.6 \\
\hline Enzyme regulator activity & 2.3 & 1.4 \\
\hline Metallochaperone activity & 0.1 & 0.1 \\
\hline Molecular transducer activity & 0.0 & 1.1 \\
\hline Motor activity & 0.4 & 0.5 \\
\hline Structural molecule activity & 14.5 & 12.4 \\
\hline Transcription regulator activity & 2.2 & 1.4 \\
\hline Translation regulator activity & 2.3 & 1.1 \\
\hline Transporter activity & 8.8 & 8.9
\end{tabular}


Based on molecular function, gene ontologies, peptidase activity (GO:0008233, $P=0.00247923$ ), catalytic activity (GO:0003824, $P=0.00488696$ ), and endopeptidase activity (GO:0004175, $P=0.00597939$ ) were found significantly upregulated in blood-fed infected condition (Figure 2). In biological process ontologies such as digestion (GO:0007586), proteolysis (GO:0006508), cellular lipid metabolic process (GO:0044255), electron transport (GO:0006118), and acyl-CoA metabolic process (GO:0006637) were found overrepresented upon blood feeding (details in Additional file 3). We also identified many dominant and differentially expressed transcripts in the two cDNA libraries indicating their prominent role in the stage specific physiological and/or biochemical processes in the mosquito vector. Based on IDEG6 analysis of EST data, 114 BLASTX-annotated genes were differentially regulated upon sugar feeding and parasite infection with blood ingestion. Sugar-fed condition exhibited a significant upregulation in the expression of many ribosomal proteins, mitochondrial proteins and other housekeeping genes $(P<0.05$, Additional file 4$)$. Dominance of proteases like various isoforms of trypsin, chymotrypsin precursors, carboxypeptidases, and other proteases $(P<0.05)$ characterized the blood-fed infected female mosquitoes as reported earlier $[17,36,37]$. However, some isoforms of serine proteases were also significantly overexpressed in the sugar-fed tissue. We also identified 5 unannotated genes (PU_Contig136 ( $P \leq 0.05)$, PU_Contig398 ( $P \leq$ 0.05), PU_Contig398 $(P \leq 0.05)$, PU_Contig33 \& PI_Contig478 $(P \leq 0.05)$, and PU_Contig9 \& PI_Contig24 $(P \leq 0.05))$, which were significantly altered during the two conditions. These require further characterization (Additional file 4).

Encountering antimicrobial peptides like cecropin-A precursor $(P=0.014491)$ and cecropin-B precursor $(P=0)$ with a prominent dominance in sugar-fed condition is a unique observation. It is noteworthy that cecropins are the first reported antimicrobial peptides from insects [38], also known to have antiparasitic activity in mosquitoes [39]. However, the other well known antimicrobial peptide, defensins, with characteristic six cysteine/three disulfide bridge pattern $[40,41]$, showed no differential expression $(P>0.05)$ between the two conditions. Defensins are primarily active against gram-positive bacteria and are induced by Plasmodium or other microbial infections in mosquitoes [42]. Another transcript encoding an uncharacterized immune response-related protein [GenBank: EX221808] $(P=0.044556)$ was overexpressed upon sugar feeding. Lysozyme C-7 and salivary lysozyme (homologous to Lysozyme C-1 from A. gambiae) transcripts were also expressed in the sugar-fed tissue $(P>$ $0.05)$. These molecules participate in innate immunity [43] by catalyzing hydrolysis of the peptidoglycan layer of bacterial cell wall.
Table 5: Percent distribution of UTs among the assigned GO terms based on (A) Biological processes, (B) Molecular function, and $(C)$ Cellular component. (Continued)

C) Cellular Component

\begin{tabular}{lcc}
\hline Apical part of cell & 0.1 & 0.3 \\
\hline Extracellular space & 0.1 & 0.1 \\
\hline Intercellular bridge & 0.2 & 0.2 \\
\hline Intracellular & 30.8 & 22.6 \\
\hline Intracellular organelle & 21.5 & 19.3 \\
\hline Membrane & 10.7 & 9.4 \\
\hline Membrane-bound organelle & 8.7 & 13.3 \\
\hline Non-membrane-bound organelle & 5.7 & 7.9 \\
\hline Organelle lumen & 1.4 & 2.9 \\
\hline Organelle membrane & 5.9 & 5.9 \\
\hline Protein complex & 9.8 & 11.1 \\
\hline Ribonucleoprotein complex & 4.6 & 6.6 \\
\hline Vesicle & 0.3 & 0.3 \\
\hline Viral & & 0.0 \\
\hline
\end{tabular}

Blood feeding causes excess protein and iron overload in mosquitoes [44]. Blood-induced expression of protease transcripts would therefore be expected $[17,36,37]$. These proteolytic enzymes not only help in protein digestion but also facilitate establishment of parasite infection through proteolytic activation of enzymes, e.g., conversion of pro-chitinase to chitinase in Plasmodium gallinaceum, which digests the peritrophic matrix [45]. Postiron overdose caused by blood feeding also induces synthesis and secretion of iron storing molecules like ferritin, which defend mosquito cells from iron toxicity [46]. In our study, increased expression of putative ferritin transcripts in the blood-fed tissue, e.g., ferritin subunit 1 and secreted ferritin G subunit $(P<0.05$, Additional file 4$)$ substantiated this fact. Transcripts encoding Protein G12 precursor were exclusively ( $\mathrm{n}=335, P=0$ ) seen in the blood-fed tissue as reported earlier [23]. This protein shows homology with Bla g1 and Per a1, allergens from cockroaches, which are shed in the insect feces and upon inhalation these cause asthma in human beings [47]. The other protein G12 counterparts, ANG12 from A. gambiae [23] and AEG12 from Ae. aegypti [48] are both induced upon blood feeding. Interestingly, AEG12 is postulated to 
have a function in digestion and it maps to a genomic region affecting susceptibility to parasite infection [49].

Serpins are serine protease inhibitors, deriving their name from their activity [50]. Many studies have identified different genes and isoforms of serpins in A. gambiae $[17,51,52]$. In A. gambiae, Serpin 2 (SRPN2) is reported to negatively regulate ookinete killing and melanization thereby assisting midgut invasion by malaria parasites [52]. Encountering this transcript [GenBank: EX215382] $(P>0.05)$ in blood-fed infected tissue corroborates this fact.

Mosquito and Plasmodium chitinases are shown to promote successful establishment of the parasite by digesting the midgut peritrophic matrix [53-55]. Chitinase expression is reported to increase upon bacterial and pathogen infection [45]. A similar increase in chitinase expression $(P=0.008282)$ in the ookinete-infected tissue substantiates the fact.

As reported earlier, parasite invasion in mosquito midgut epithelia induces a cascade of changes leading to cell death by apoptosis [56]. In the blood-fed infected tissue, we also observed expression of apoptotic transcripts like caspase- 6 and ancaspase-7 [23]. Transcripts encoding anti-apoptotic proteins, which modulate caspase activity were found to be expressed in sugar-fed mosquito tissues, e.g., defender against programmed cell death. In bloodfed infected tissue, we also observed an increase in the

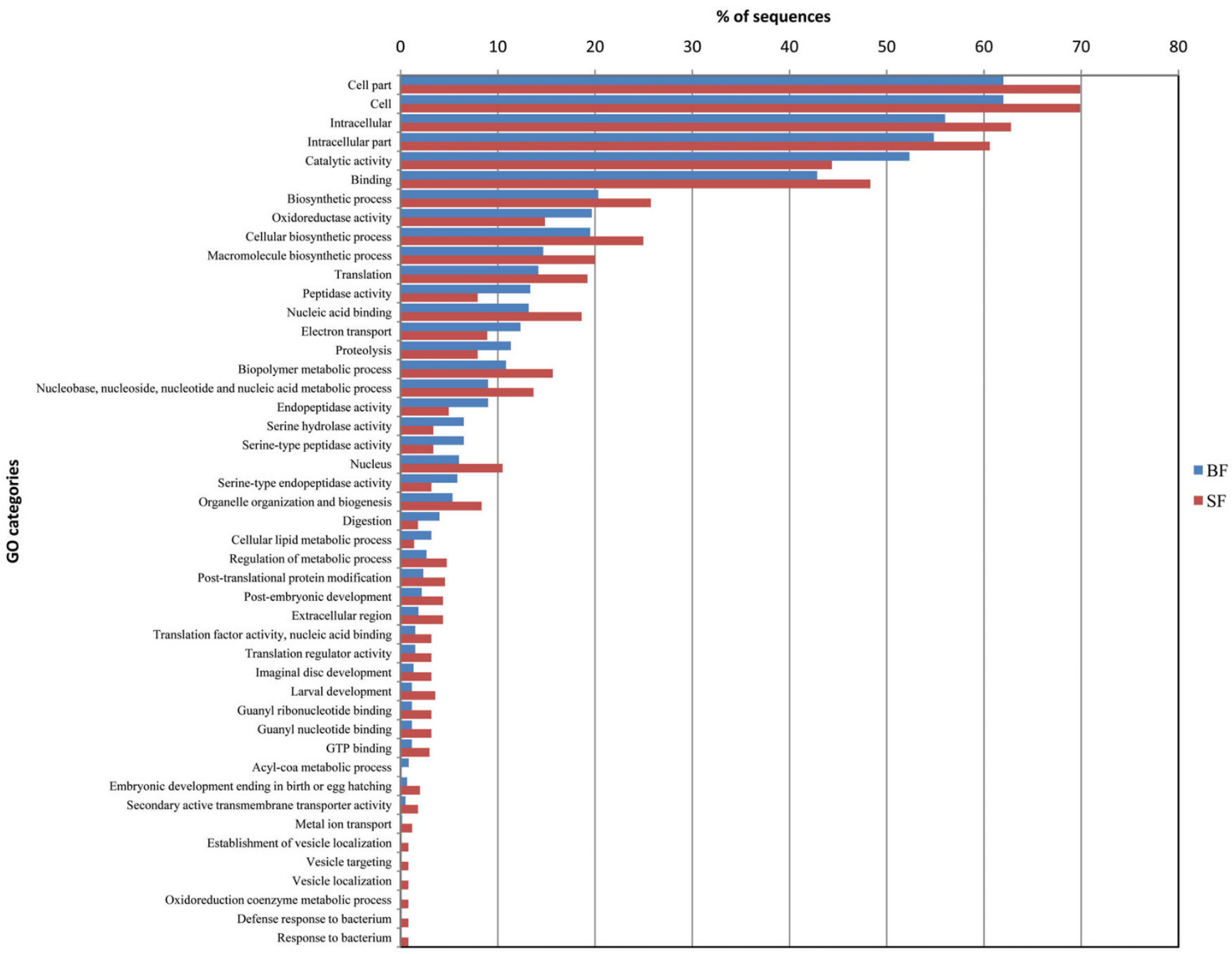

\section{Figure 2}

Statistical comparison of GO terms assigned to UTs from SF and BF libraries. The figure shows comparison of GO terms assigned to annotated UTs from SF and BF libraries based on Fisher's exact test. Only significantly altered $(P<0.05) \mathrm{GO}$ terms are shown here. Other details are provided in Additional file 3. Note that individual GO terms can be assigned to multiple UTs and one UT can have multiple GO terms. 
Table 6: Summary of $A$. stephensi UTs mapped on $A$. gambiae chromosomes using Gmap program with default parameters.

\begin{tabular}{ccc}
\hline A. gambiae chromosome & \multicolumn{2}{c}{ Combined } \\
\cline { 2 - 3 } & Singlets & Contigs \\
\hline $2 \mathrm{~L}$ & 137 & 154 \\
\hline $2 \mathrm{R}$ & 237 & 177 \\
\hline $3 \mathrm{~L}$ & 121 & 121 \\
\hline $3 \mathrm{R}$ & 163 & 151 \\
\hline $\mathrm{X}$ & 44 & 44 \\
\hline Unknown & 17 & 21 \\
\hline Y unplaced & - & - \\
\hline Total & 719 & 668
\end{tabular}

number of several enzymes participating in redox metabolism and detoxification, such as superoxide dismutase, peroxidase, isoforms of metallothionein, cytochrome P450, and glutathione-S-transferase (Additional file 4). Some of the oxidoreductases were found in both the libraries but an overall upregulation is evident upon blood feeding and parasite infection, as reported earlier $[57,58]$.

Cytoskeletal remodeling in host cells is a hallmark of pathogen attachment and invasion during infection [5961]. We also found many transcripts encoding cytoskeletal and its associated proteins during both the conditions. As the regulation of formation of the actin network in cell cytoskeleton is centered at Arp2/3 complex (ARP) [62], its overexpression is necessary during infection. A significant increase in ARPs, $\alpha$ and $\beta$ tubulins are reported to be upregulated during parasite invasion in A. gambiae midgut [63]. However, we did not find such difference. Pathogen establishment is a stress to the host cell [64] accompanied with oxidative burst leading to misfolding of proteins $[65,66]$. A vast variety of stress induced proteins, especially, heat shock proteins and chaperonins are produced by the cell to carry out proper protein folding during stress. Many such transcripts were also observed in our data (Additional file 1).

Tetraspanins are conserved membrane proteins traversing cell membrane four times [67]. These are found associated with many other proteins, especially integrins. They are involved in intracellular signaling, cellular motility, and metastasis. We found tetraspanin transcripts (Additional file 1) in both conditions. In Drosophila [67], the tet- raspanin family comprises more than 30 members suggesting a possibility of many such proteins in mosquitoes. Interestingly, in Manduca sexta, tetraspanin-integrin interactions have been reported necessary for transition of hemocytes during cell-mediated immune responses [68].

Proteins containing leucine-rich repeats (LRRs) like APL1 [12], LRIM1, and LRIM2 [69] demonstrate inhibitory activity against Plasmodium infection in A. gambiae and Anopheles quadriannulatus [70]. Many other LRR domain containing proteins like toll receptors are reported in insects and other organisms, which primarily participate in protein-protein interactions [53]. They exhibit diverse functionality but a definitive role has not been established in insects. We also found a few transcripts encoding proteins with LRR domains in our study (Additional file 1).

ICHIT protein contains mucin domains, which participate in the formation of extracellular matrix [71], and in trapping microbial pathogens through their lectin-liking characteristics [72]. It possesses two putative chitin-binding domains flanking a mucin domain, and is observed to increase upon bacterial and malaria challenge in A. gambiae [73]. However, we observed an increase in ICHIT $(P=$ $0.000019)$ transcripts in sugar-fed condition. This protein is also believed to be associated with the peritrophic matrix, which separates the blood meal from the midgut membrane. Found across many other organisms, a possible role of ICHIT in immune response is predicted against pathogens [71].

Septins are GTPases thought to be associated with cell division especially nuclear division, membrane trafficking, and organizing the cytoskeleton [74]. As in other studies [37], we also observed septins and smt 3 transcripts in the sugar-fed tissue. These together play a role in toll signaling [37]. We found many other insignificantly expressed transcripts in both the conditions (Additional file 4), which might bear an indispensable role in mosquito life cycle, e.g., vitellogenin, which is an abundant yolk precursor protein participating in egg maturation [75].

In summary, our study identifies numerous transcripts from A. stephensi midgut tissue with known and unknown functions (Additional file 1). However, despite of massive sequencing, loss of rare transcripts is possible. This could be due to the overexpression of certain stage specific genes, e.g., blood-induced genes like trypsin. In addition, our study differs with respect to the use of incubation temperature $\left(28^{\circ} \mathrm{C}\right)$ for parasite development in mosquitoes from work reported earlier $\left(24^{\circ} \mathrm{C}\right)$ [76]. However, we observed reasonable number of oocyst formation (average $65.3, \mathrm{n}=20$ ). Blood feeding by these parasite-carrying mosquitoes also induced a significant parasitemia in 
uninfected mice, confirming completion of parasite life cycle in the vector at $28^{\circ} \mathrm{C}$. Furthermore, in the view of low genomic and proteomic resemblance between $P$. yoelii and the human malaria parasites [77], observations from rodent models like ours, need an essential analysis and assessment before extrapolation. Nevertheless, the information generated in the form of transcriptome could certainly prove a boon in investigating other malaria parasites.

\section{Conclusion}

We have successfully obtained 3946 transcripts from the adult female A. stephensi mosquito midgut, which would be of considerable use in future research on this malaria vector. Mapping of transcripts onto the A. gambiae genome was beneficial in the gene discovery process.

\section{Methods}

\section{In vivo maintenance of parasites}

Plasmodium parasites (P. yoelii) were obtained from the Malaria Research Center (Delhi, India). The parasite was first inoculated in adult BALB/c mice (UK/AIIMS strains) by intraperitoneal route. Time for effective parasitemia was determined on various post inoculation days (PID). Parasites were maintained in vivo throughout the study.

\section{Mosquito rearing and Parasite infection}

A. stephensi (NIV strain) mosquitoes were maintained on $10 \%$ glucose until blood feeding. Adult females (4 days old) were allowed to blood feed on $P$. yoelii infectedBALB/c mice. Prior to blood feeding, blood parasitemia levels in the infected mice were determined using Giemsa stain. Mice showing gametocyte percentages above 0.5 were used for blood feeding experiments as reported earlier [78]. Fully engorged females were separated using an aspirator and maintained in the insectory with controlled temperature $\left(28 \pm 2^{\circ} \mathrm{C}\right)$ and humidity $(80 \pm 5 \%)$ under 12 $\mathrm{h}$ alternating dark/light cycles. At $24 \mathrm{~h}$ post blood feeding, mosquito midguts were dissected, stained with $0.5 \%$ mercurochrome, and oocyst numbers per midgut were determined using a light-contrast microscope (Olympus) at $100 \times$ magnification. Dissected midguts were stored in liquid nitrogen until cDNA library preparation. To confirm completion of Plasmodium sporogony cycle in mosquitoes at $28^{\circ} \mathrm{C}$, after every $4^{\text {th }}$ or $5^{\text {th }}$ passage, the natural route of infection was confirmed i.e. parasite-infected mosquitoes (14-15 days post blood feeding) were allowed to feed on uninfected BALB/c mice and parasitemia was recorded. To determine ookinete infection in the infected tissue, we additionally performed a qualitative assay based on reverse transcriptase PCR (RT-PCR) for $P$. yoelii ookinete specific genes, pyCTRP [79] and pyECP1 [80] (for details refer Additional file 2).

\section{RNA extraction, cDNA library preparation and DNA sequencing}

A set of 20-40 blood-fed infected and sugar-fed adult female midgut tissues were used for cDNA library preparation. The tissues were crushed in trizol (Invitrogen) using RNase-free glass dounce homogenizer. RNA was subsequently extracted, following the manufacturer's protocol. Quantification of RNA was performed using ND1000 Nanodrop (Thermo Scientific). RNA integrity was checked using denaturing agarose gel electrophoresis. cDNA libraries were constructed using the Creator ${ }^{\mathrm{TM}}$

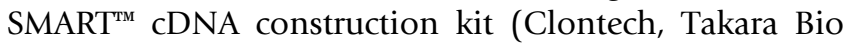
Inc.) according to the manufacturer's protocol using $1 \mu \mathrm{g}$ of total RNA. After digestion with Sfi I, cDNA fragments were size fractionated using CHROMA SPIN-400 columns according to the instructions provided. Fractions were checked on $1.5 \%$ agarose/EtBr gels. cDNA fragments ranging from $300 \mathrm{bp}$ to $3 \mathrm{~kb}$ were pooled. All further steps including ligation to pDNR-LIB, precipitation, and electroporation (Biorad GenePulser) in DH10B E. coli (Invitrogen) were carried out following the supplier's instructions. Libraries were screened for inserts by colony PCR. Thereafter, primary libraries were amplified and stored in $25 \%$ glycerol stocks at $-80^{\circ} \mathrm{C}$. When required, clones were plated using LB (Luria-Bertani) agar containing $30 \mu \mathrm{g} / \mathrm{ml}$ chloramphenicol and incubated overnight at $37^{\circ} \mathrm{C}$. Colonies were manually inoculated in $1 \mathrm{ml} 2 \times$ LB broth containing chloramphenicol in a 96-well inoculation plate. Plasmid isolation was done using Montage Plasmid Miniprep ${ }_{96}$ kit (LSKP09624, Millipore Corporation) following the manufacturer's instructions. Plasmid concentrations were determined for a random set of clones from each 96-well plate using nanodrop and quality was checked on 1\% agarose/EtBr gels. Approximately, 300-500 ng of plasmids containing cDNA insert were sequenced from their 5 ' end using BigDye Terminator version 3.1 chemistry (Applied Biosystems, Foster City, CA) and M13 primer (5'-GTAAAACGACGGCCAGTAGATCT$3^{\prime}$ ) on an ABI 3730 Genetic analyzer (Applied Biosystems) following the manufacturer's protocol.

\section{Bioinformatics Analysis}

The EST analysis was performed using an in-house developed EST pipeline (Additional file 2). Base-calling of the trace files was performed using phred [25,26] (quality value $\geq 20$ ). The vector, primer and adapter sequences were masked using cross_match. PolyA tails were removed using a program in PERL script. Trimmed ESTs less than 100 bases in length were discarded. An additional round of filtering was performed to remove vector sequence, adapter sequence, and polyA tail using seqclean [81]. EST sequences representing mouse and Plasmodium genes were identified and removed using BLAST analysis. ESTs from BF and SF libraries were assembled separately and together using CAP3 program [29]. The UTs (contigs 
plus singlets) obtained from both libraries were combined and assembled using CAP3. These were searched against the UniProtKB database using BLASTX and EST data of A. gambiae, Ae. aegypti, and D. melanogaster, using TBLASTX. UTs showing no significant hits with the UniProtKB database were scanned using ESTScan [31] to verify the presence of putative coding region. GO terms were assigned to all the UTs using Blast2GO program [32]. Classifications were based on molecular function, biological processes, and cellular components. To identify overrepresented GO terms between the libraries, enrichment analysis (using Fisher's exact test at a significance threshold value of 0.05) was carried out in Blast2GO program.

\section{Differential gene expression-IDEG6 Analysis}

Statistical comparison of gene expression in both the libraries was performed using the online version of IDEG6 [34] implementing pairwise Fisher exact test (significance threshold of 0.05). The analysis was performed for BLASTX-annotated and unannotated ESTs separately. For unannotated ESTs, only ESTs containing a putative coding region were considered.

\section{Genome mapping}

Files representing the $2 \mathrm{~L}, 2 \mathrm{R}, 3 \mathrm{R}, 3 \mathrm{~L}, \mathrm{X}$, unknown, and unplaced $\mathrm{Y}$ chromosomal sequences were downloaded from Ensembl [82]. UTs were mapped onto the A. gambiae genome using Gmap version 2007-09-28 [83] using default parameters. Information comprising number of exons, chromosome name, and locus, was parsed using PERL script.

\section{Development of ESTDB}

MySQL relational database management system was used as the back-end and the front-end was designed using various modules of PERL (CGI, DBI and GD). The database is hosted on the web using Apache web-server.

\section{Accession numbers}

All the ESTs were deposited in the GenBank database with accession numbers from EX212289 to EX227655.

\section{List of abbreviations used (if any)}

ESTs: Expressed Sequence Tags; BF: Plasmodium yoelii infected blood-fed; SF: Sugar-fed; UTRs: untranslated regions; PERL: Practical Extraction and Reporting Language; and UTs: Unique transcripts (refers to singlets and contigs together).

\section{Authors' contributions}

DPP, DPD, and AD were involved in construction of cDNA libraries. DPP, VSM, SAW, RKC, GJK, PSG, AS, and KMD contributed in sequencing the libraries. SA designed the pipeline for EST sequence trimming, assembly, and construction of ESTDB database. ESTscan and UniprotKB
BLAST analyses were performed by SA with the help of BB. DPD has performed GO analysis, insect-specific transcript identification, IDEG6 analysis, and mapping of ESTs on A. gambiae genome with the help of DPP \& NG. DTM performed mosquito rearing, parasite maintenance, and parasite infection in mosquitoes. DTM, RRJ, and MSP were co-investigators with YSS. DPP and DPD were involved in preparing the manuscript. All authors read and approved the final manuscript.

\section{Additional material}

\begin{abstract}
Additional file 1
Detailed report of EST analysis. Contains detailed information obtained for all transcripts by various analyses. The worksheet includes user ID, GenBank accession, library-specificity (SF/BF/Both), insect-specific (YES/NO), insect-specific protein ID (if present), genome mapping to A. gambiae ('YES', if mapped and 'No', if not mapped), BLAST results (e.g., sequence description, length of query sequence, no. of BLAST hits obtained (maximum 10), maximum E-value, mean similarity (average similarity of top 10 BLAST hits)), no. of GO IDs, GO IDs, EC No., and other genome mapping details.

Click here for file

[http://www.biomedcentral.com/content/supplementary/14712164-10-386-S1.xls]
\end{abstract}

\section{Additional file 2}

Assessment of Plasmodium infection in mosquito midgut and additional figures. Contains protocol for PCR based assessment of Plasmodium yeolii ookinete infection in the fermale A. stephensi mosquito midgut and results (Additional file 2: Figure S1). E-value-, percent-, and similarity-distribution for $S F, B F$, and combined UTs (Additional file 2: Figure S2). Flow chart depicting flow of analysis for EST pre-processing and functional annotation (Additional file 2: Figure S3).

Click here for file

[http://www.biomedcentral.com/content/supplementary/14712164-10-386-S2.pdf]

\section{Additional file 3}

Statistical comparison of GO terms. The file contains detailed output of Blast2GO's Enrichment analysis based on Fisher's exact test (only significantly $(\mathrm{P}<0.05)$ altered $\mathrm{GO}$ term representations are shown).

Click here for file

[http://www.biomedcentral.com/content/supplementary/14712164-10-386-S3.xls]

\section{Additional file 4}

Statistical comparison for differential gene expression (IDEG6 analysis). The file contains statistical comparison of the genes expressed in both the libraries using IDEG6 tool. Comparison of annotated and unannotated genes is given in "Annotated" and "Unannotated" worksheets, respectively. Different degrees of blue shades in the normalized tag values represent the extent of gene expression. Significant P-values are shaded yellow.

Click here for file

[http://www.biomedcentral.com/content/supplementary/14712164-10-386-S4.xls] 


\section{Acknowledgements}

We are truly grateful to Prof. David W. Severson (Department of Biological Sciences, University of Notre Dame, USA), Dr. R. N. Sharma (National Chemical Laboratory, Pune, India), and Dr. Kiran Pawar (NCCS) for their comments and critical review of the manuscript. We thank the Department of Biotechnology, Government of India for providing financial support to the project. We also thank the Council of Scientific and Industrial Research (CSIR, New Delhi, India) and Department of Biotechnology (New Delhi, India) for supporting DPP and DPD with fellowships, respectively. We express our gratitude towards Mr. Sarang Satoor, Incharge, DNA Sequencing Facility, NCCS, for his kind help and suggestions in sequencing. We gratefully acknowledge the active support and encouragement provided by the Director, NCCS, Padmashree, Dr. G. C. Mishra.

\section{References}

I. Oshaghi MA, Yaaghoobi F, Abaie MR: Pattern of mitochondrial DNA variation between and within Anopheles stephensi (Diptera: Culicidae) biological forms suggests extensive gene flow. Acta Trop 2006, 99:226-233.

2. Dash AP, Adak T, Raghavendra K, Singh OP: The biology and control of malaria vectors in India. Curr Sci 2007, 92:157|- 1578.

3. Chatterjee $P$ : India faces new challenges in the fight against malaria. Lancet Infect Dis 2006, 6:324.

4. Moorthy VS, Good MF, Hill AV: Malaria vaccine developments. Lancet 2004, 363:150-156.

5. Hyde JE: Drug-resistant malaria. Trends Parasitol 2005, 21:494-498.

6. Kumar N: A vaccine to prevent transmission of human malaria: A long way to travel on a dusty and often bumpy road. Curr Sci 2007, 92:1535-1544.

7. Porter AG: Mosquitocidal toxins, genes and bacteria: the hit squad. Parasitol Today 1996, 12:175-179.

8. Blanford S, Chan BH, Jenkins N, Sim D, Turner RJ, Read AF, Thomas MB: Fungal pathogen reduces potential for malaria transmission. Science 2005, 308:1638-164I.

9. Ito J, Ghosh A, Moreira LA, Wimmer EA, Jacobs-Lorena M: Transgenic anopheline mosquitoes impaired in transmission of a malaria parasite. Nature 2002, 4I7:452-455.

10. Boete C: Malaria parasites in mosquitoes: laboratory models, evolutionary temptation and the real world. Trends Parasitol 2005, 21:445-447.

II. Cohuet A, Osta MA, Morlais I, Awono-Ambene PH, Michel K, Simard F, Christophides GK, Fontenille D, Kafatos FC: Anopheles and Plasmodium: from laboratory models to natural systems in the field. EMBO Rep 2006, 7:1285-1289.

12. Riehle MM, Markianos K, Niare O, Xu J, Li J, Toure AM, Podiougou B, Oduol F, Diawara S, Diallo M, et al: Natural malaria infection in Anopheles gambiae is regulated by a single genomic control region. Science 2006, 3 I 2:577-579.

13. Abraham EG, Pinto SB, Ghosh A, Vanlandingham DL, Budd A, Higgs $\mathrm{S}$, Kafatos FC, Jacobs-Lorena M, Michel K: An immune-responsive serpin, SRPN6, mediates mosquito defense against malaria parasites. Proc Natl Acad Sci USA 2005, 102:16327-16332.

14. Blandin S, Shiao SH, Moita LF, Janse CJ, Waters AP, Kafatos FC, Levashina EA: Complement-like protein TEPI is a determinant of vectorial capacity in the malaria vector Anopheles gambiae. Cell 2004, I 16:661-670.

15. Osta MA, Christophides GK, Kafatos FC: Effects of mosquito genes on Plasmodium development. Science 2004, 303:2030-2032.

16. Holt RA, Subramanian GM, Halpern A, Sutton GG, Charlab R, Nusskern DR, Wincker P, Clark AG, Ribeiro JM, Wides R, et al:: The genome sequence of the malaria mosquito Anopheles gambiae. Science 2002, 298: 129-149.

17. Ribeiro JM: A catalogue of Anopheles gambiae transcripts significantly more or less expressed following a blood meal. Insect Biochem Mol Biol 2003, 33:865-82.

18. Gomez SM, Eiglmeier K, Segurens B, Dehoux P, Couloux A, Scarpelli C, Wincker P, Weissenbach J, Brey PT, Roth CW: Pilot Anopheles gambiae full-length cDNA study: sequencing and initial characterization of 35,575 clones. Genome Biol 2005, 6: I-R39.

19. Dimopoulos G, Casavant TL, Chang S, Scheetz T, Roberts C, Donohue M, Schultz H, Benes V, Bork P, Ansorge W, Soares Mb, Kafatos
FC: Anopheles gambiae pilot gene discovery project: Identification of mosquito innate immunity genes from expressed sequence tags generated from immune-competent cell lines. Proc Natl Acad Sci USA 2000, 97:6619-6624.

20. Calvo E, Pham VM, Lombardo F, Arca B, Ribeiro JM: The sialotranscriptome of adult male Anopheles gambiae mosquitoes. Insect Biochem Mol Biol 2006, 36:570-575.

21. Dimopoulos G, Christophides GK, Meister S, Schultz J, White KP, Mury CB, Kafatos FC: Genome expression analysis of Anopheles gambiae: Responses to injury, bacterial challenge, and malaria infection. Proc Natl Acad Sci USA 2002, 99:88I4-88I9.

22. Valenzuela JG, Francischetti IM, Pham VM, Garfield MK, Ribeiro JM: Exploring the salivary gland transcriptome and proteome of the Anopheles stephensi mosquito. Insect Biochem Mol Biol 2003, 33:717-732

23. Abraham EG, Islam S, Srinivasan $P$, Ghosh AK, Valenzuela JG, Ribeiro JM, Kafatos FC, Dimopoulos G, Jacobs-Lorena M: Analysis of the Plasmodium and Anopheles transcriptional repertoire during ookinete development and midgut invasion. J Biol Chem 2004, 279:5573-5580.

24. Wakaguri H, Suzuki Y, Katayama T, Kawashima S, Kibukawa E, Hiranuka K, Sasaki M, Sugano S, Watanabe J: Full-Malaria/Parasites and Full-Arthropods:databases of full-length cDNAs of parasites and arthropods, update 2009. Nucleic Acid Research 2009, 37:D520-D525.

25. Xu X, Dong Y, Abraham EG, Kocan A, Srinivasan P, Ghosh AK, Sinden RE, Ribeiro JM, Jacobs-Lorena M, Kafatos FC, et al.: Transcriptome analysis of Anopheles stephensi - Plasmodium berghei interactions. Mol Biochem Parasitol 2005, I 42:76-87.

26. Ewing B, Hillier L, Wendl MC, Green P: Base-calling of automated sequencer traces using phred. I. Accuracy assessment. Genome Res 1998, 8:175-185.

27. Ewing B, Green P: Base-calling of automated sequencer traces using phred. II. Error probabilities. Genome Res 1998, 8:186-194.

28. Phred, Phrap, Consed [http://www.phrap.org]

29. Huang $X$, Madan A: CAP3: A DNA sequence assembly program. Genome Res 1999, 9:868-877.

30. Nagaraj SH, Gasser RB, Ranganathan S: A hitchhiker's guide to expressed sequence tag (EST) analysis. Brief Bioinform 2007, 8:6-21.

31. Iseli C, Jongeneel CV, Bucher P: ESTScan: a program for detecting, evaluating, and reconstructing potential coding regions in EST sequences. ISMB 1999: I38-148.

32. Conesa A, Gotz S, Garcia-Gomez JM, Terol J, Talon M, Robles M: Blast2GO: a universal tool for annotation, visualization and analysis in functional genomics research. Bioinformatics 2005 , 21:3674-3676.

33. Ashburner M, Ball CA, Blake JA, Botstein D, Butler H, Cherry JM, Davis AP, Dolinski K, Dwight SS, Eppig JT, et al.: Gene Ontology: tool for the unification of biology. Nat Genetics 2000, 25:25-29.

34. Romualdi C, Bortoluzzi S, D'Alessi F, Danieli GA: IDEG6: a web tool for detection of differentially expressed genes in multiple tag sampling experiments. Physiol Genomics 2003, 12:159-162.

35. Zhang G, Wang $\mathrm{H}$, Shi J, Wang $\mathrm{X}$, Zheng $\mathrm{H}$, Wong $\mathrm{G}$, Clark T, Wang $W$, Wang J, Kang $L$ : Identification and characterization of insect-specific proteins by genome data analysis. BMC Genomics 2007, 8:93-104.

36. Dana AN, Hong YS, Kern MK, Hillenmeyer ME, Harker BW, Lobo $\mathrm{NF}$, Hogan JR, Romans $\mathrm{P}$, Collins $\mathrm{FH}$ : Gene expression patterns associated with blood-feeding in the malaria mosquito Anopheles gambiae. BMC Genomics 2005, 6:5-29.

37. Dana AN, Hillenmeyer ME, Lobo NF, Kern MK, Romans PA, Collins $\mathrm{FH}$ : Differential gene expression in abdomens of the malaria vector mosquito, Anopheles gambiae, after sugar feeding, blood feeding and Plasmodium berghei infection. BMC Genomics 2006, 7:119.

38. Bulet P, Hetru C, Dimarcq JL, Hoffmann D: Antimicrobial peptides in insects; structure and function. Dev Comp Immunol 1999, 23:329-344.

39. Vizioli J, Bulet P, Charlet M, Lowenberger C, Blass C, Muller HM, Dimopoulos G, Hoffmann J, Kafatos FC, Richman A: Cloning and analysis of a cecropin gene from the malaria vector mosquito, Anopheles gambiae. Insect Mol Biol 2000, 9:75-84. 
40. Rees JA, Moniatte M, Bulet P: Novel antibacterial peptides isolated from a European bumblebee, Bombus pascuorum (Hymenoptera, Apoidea). Insect Biochem Mol Biol 1997, 27:413-422.

41. Rossignol PA, Lueders AM: Bacteriolytic factor in the salivary glands of Aedes aegypti. Comp Biochem Physiol B 1986, 83:8I 9-822.

42. Dimopoulos G, Richman A, Muller HM, Kafatos FC: Molecular immune responses of the mosquito Anopheles gambiae to bacteria and malaria parasites. Proc Natl Acad Sci USA 1997 94:II508-II5I3

43. Schmid-Hempel P: Evolutionary ecology of insect immune defenses. Annu Rev Entomol 2005, 50:529-55I.

44. Lehane MJ: The Biology of Blood-Sucking in Insects Cambridge: Cambridge University Press; 2005.

45. Sinden RE: Molecular interactions between Plasmodium and its insect vectors. Cell Microbiol 2002, 4:7I 3-724.

46. Geiser DL, Zhang D, Winzerling J]: Secreted ferritin: mosquito defense against iron overload? Insect Biochem Mol Biol 2006 36: $177-187$

47. Pomes A, Melen E, Vailes LD, Retief JD, Arruda LK, Chapman MD: Novel allergen structures with tandem amino acid repeats derived from German and American cockroach. J Biol Chem 1998, 273:3080|-30807.

48. Shao L, Devenport M, Fujioka H, Ghosh A, Jacobs-Lorena M: Identification and characterization of a novel peritrophic matrix protein, Ae-Aper50, and the microvillar membrane protein, AEG I 2, from the mosquito, Aedes aegypti. Insect Biochem Mol Biol 2005, 35:947-959.

49. Morlais I, Mori A, Schneider JR, Severson DW: A targeted approach to the identification of candidate genes determining susceptibility to Plasmodium gallinaceum in Aedes aegypti. Mol Genet Genomics 2003, 269:753-764.

50. Carrell R, Travis J: $\alpha$ I-Antitrypsin and the serpins: Variation and coutervariation. Trends Biochem Sci 1985, 10:20-24.

51. Danielli A, Kafatos FC, Loukeris TG: Cloning and characterization of four Anopheles gambiae serpin isoforms, differentially induced in the midgut by Plasmodium berghei invasion. J Biol Chem 2003, 278:4184-4I93.

52. Michel K, Budd A, Pinto S, Gibson TJ, Kafatos FC: Anopheles gambiae SRPN2 facilitates midgut invasion by the malaria parasite Plasmodium berghei. EMBO Rep 2005, 6:89I-897.

53. Kobe $B, K$ ajava $A V$ : The leucine-rich repeat as a protein recognition motif. Curr Opin Struct Biol 200 I, I I:725-732.

54. Huber M, Cabib E, Miller LH: Malaria parasite chitinase and penetration of the mosquito peritrophic membrane. Proc Nat Acad Sci USA I99I, 88:2807-28I0.

55. Shen Z, Jacobs-Lorena M: Characterization of a novel gut-specific chitinase gene from the human malaria vector Anopheles gambiae. J Biol Chem 1997, 272:28895-28900.

56. Han YS, Thompson J, Kafatos FC, Barillas-Mury C: Molecular interactions between Anopheles stephensi midgut cells and Plasmodium berghei: the time bomb theory of ookinete invasion of mosquitoes. EMBO J 2000, 19:6030-6040.

57. Kumar S, Gupta L, Han YS, Barillas-Mury C: Inducible peroxidases mediate nitration of anopheles midgut cells undergoing apoptosis in response to Plasmodium invasion. I Biol Chem 2004, 279:53475-53482.

58. Kumar S, Christophides GK, Cantera R, Charles B, Han YS, Meister $\mathrm{S}$, Dimopoulos G, Kafatos FC, Barillas-Mury C: The role of reactive oxygen species on Plasmodium melanotic encapsulation in Anopheles gambiae. Proc Natl Acad Sci USA 2003, 100:14139-14144

59. Patel JC, Galan JE: Manipulation of the host actin cytoskeleton by Salmonella-all in the name of entry. Curr Opin Microbiol 2005, 8: $10-15$

60. Forney JR, DeWald DB, Yang S, Speer CA, Healey MC: A role for host phosphoinositide 3-kinase and cytoskeletal remodeling during Cryptosporidium parvum infection. Infect Immun 1999 , 67:844-852.

6I. Carabeo RA, Grieshaber SS, Fischer E, Hackstadt T: Chlamydia trachomatis induces remodeling of the actin cytoskeleton during attachment and entry into HeLa cells. Infect Immun 2002, 70:3793-3803.

62. Pollard TD, Borisy GG: Cellular motility driven by assembly and disassembly of actin filaments. Cell 2003, I I 2:453-465.
63. Vlachou D, Schlegelmilch T, Christophides GK, Kafatos FC: Functional genomic analysis of midgut epithelial responses in Anopheles during Plasmodium invasion. Curr Biol 2005, 15:1185-1195.

64. Larson SJ, Dunn AJ: Behavioural mechanisms for defence against pathogens. Neuroimmune Biol 2005, 5:35I-368.

65. Banhegyi G, Benedetti A, Csala M, Mandl J: Stress on redox. FEBS Lett 2007, 58 I:3634-3640.

66. Ruddock LW, Klappa P: Oxidative stress: Protein folding with a novel redox switch. Curr Biol 1999, 9:R400-R402.

67. Fradkin LG, Kamphorst JT, DiAntonio A, Goodman CS, Noordermeer JN: Genomewide analysis of the Drosophila tetraspanins reveals a subset with similar function in the formation of the embryonic synapse. Proc Natl Acad Sci USA 2002, 99:13663-13668.

68. Zhuang S, Kelo L, Nardi JB, Kanost MR: An integrin-tetraspanin interaction required for cellular innate immune responses of an insect, Manduca sexta. J Biol Chem 2007, 282:22563-22572.

69. Danielli A, Kafatos FC, Loukeris TG: Cloning and characterization of four Anopheles gambiae serpin isoforms, differentially induced in the midgut by Plasmodium berghei invasion. J Biol Chem 2003, 278:4I84-4I93.

70. Habtewold T, Povelones M, Blagborough AM, Christophides GK: Transmission blocking immunity in the malaria non-vector mosquito Anopheles quadriannulatus species A. PLoS Pathog 2008, 4:el 000070.

7I. Tellam RL, Wijfels G, Willadsen P: Peritrophic matrix proteins. Insect Biochem Mol Biol 1999, 29:87-I0I.

72. Kawabata S, Nagayama R, Hirata M, Shigenaga T, Agarwala KL, Saito T, Cho J, Nakajima H, Takagi T, Iwanaga S: Tachycitin, a small granular component in horseshoe crab hemocytes, is an antimicrobial protein with chitin-binding activity. J Biochem 1996, I 20:1253-1260.

73. Dimopoulos G, Seeley D, Wolf A, Kafatos FC: Malaria infection of the mosquito Anopheles gambiae activates immune-responsive genes during critical transition stages of the parasite life cycle. EMBO J 1998, 17:6||5-6|23.

74. Lindsey R, Momany M: Septin localization across kingdoms: three themes with variations. Curr Opin Microbiol 2006, 9:559-565.

75. Sappington TW, Raikhel AS: Molecular characteristics of insect vitellogenins and vitellogenin receptors. Insect Biochem Mol Biol 1998, 28:277-300.

76. Sinden Robert $\mathrm{E}$ : Infection of mosquitoes with rodent malaria. In The Molecular Biology of Insect Disease Vectors: A Methods Manual Edited by: Crampton JM, Beard CB, Louis C. London: Chapman \& Hall; | 1996:67-9|.

77. Hall N, Karras M, Raine JD, Carlton JM, Kooij TW, Berriman M, Florens L, Janssen CS, Pain A, Christophides GK, et al: A comprehensive survey of the Plasmodium life cycle by genomic, transcriptomic, and proteomic analyses. Science 2005, 307:82-86.

78. Blandin Stephanie A, Levashina Elena A: Reverse Genetics Analysis of Antiparasitic Responses in the Malaria Vector, Anopheles gambiae. In Innate Immunity Edited by: Jonathan Ewbank, Eric Vivier. New Jersey: Springer; 2007:365-377.

79. Kaneko O, Templeton Tj, Iriko H, Tachibana M, Otsuki H, Takeo S, Sattabongkot J, Torii M, Tsuboi T: The Plasmodium vivax homolog of the ookinete adhesive micronemal protein, CTRP. Parasitol Int 2006, 55:227-23I.

80. Aly AS, Matuschewski K: A malarial cysteine protease is necessary for Plasmodium sporozoite egress from oocysts. J Exp Med 2005, 202:225-230

8I. Pertea G, Huang X, Liang F, Antonescu V, Sultana R, Karamycheva S, Lee $Y$, White J, Cheung F, Parvizi B, et al.: TIGR Gene Indices clustering tools (TGICL): a software system for fast clustering of large EST datasets. Bioinformatics 2003, 19:65। -652.

82. Birney E, Andrews TD, Bevan P, Caccamo M, Chen Y, Clarke L, Coates G, Cuff J, Curwen V, Cutts T, et al: An overview of Ensembl. Genome Res 2004, 14:925-928.

83. Wu TD, Watanabe CK: GMAP: a genomic mapping and alignment program for mRNA and EST sequences. Bioinformatics 2005, 2 I: |859-1875 